\title{
ÜBER EINIGE FÄLLE HOHER LUFTTEMPERATUR IN STORSLETT (NORDNORWEGEN)
}

\author{
R. H. G. Mook \\ E INFÜHRUNG
}

An der synoptischen Wetterstation Storslett wurden mehrfach auffallend hohe Lufttemperaturen beobachtet. Ein systematischer Fehler in den Messungen ist nicht anzunehmen. Registrierungen liegen leider nicht vor; die Zuverlässigkeit der visuellen Temperaturablesungen muß daher vorausgesetzt werden.

Storslett liegt auf $69^{\circ}+6^{\circ} \mathrm{N}, 21^{\circ} 2^{\prime} \mathrm{E}$ und $+\mathrm{m}$ Meereshöhe, in einem weiten Talboden, der nach Norden hin zum Reisafjord offen ist. Nach Süden und Südosten ziehen das Reisa- und das Kiltal. Vorherrschende Windrichtung ist, zumal im Winter, Süd und Südost. Für andere Windrichtungen, die selten beobachtet werden, ist die Station kaum zuständig (1). Im Osten liegen in etwa $5 \mathrm{~km}$ Entfernung $1000 \mathrm{~m}$ hohe Gebirge, im Südwesten und Westen in $7 \mathrm{~km}$ bzw. $4 \mathrm{~km}$ Entfernung $1200 \mathrm{~m}$ hohe Erhebungen. Boatka mit $1050 \mathrm{~m}$ im Südosten liegt $14 \mathrm{~km}$ weit entfernt. Der Orographie nach dürften montane Föhnwirkungen möglich sein. Für seine Anregungen bin ich Herrn Staatsmeteorologe E. Wishman zu Dank verpflichtet.

\section{BEOBACHTUNGEN}

Drei ausgewählte Fälle hoher Temperatur seien im folgenden näher besprochen:

Der Temperaturrekord vom 2. Juni 1961. Am 2.6.1961 wurde in Storslett das Maximum von $35.4^{\circ} \mathrm{C}$ gemessen, die höchste im nördlichen Norwegen jemals abgelesene Temperatur. Nach Versicherung der Beobachterin Lund (2) ist die Ablesung zuverlässig, und schon wiederholt seien markante Temperaturmaxima an der Station aufgetreten. Subjektiv wurden «warme Wellen» festgestellt. Es erscheint zweifelhaft, ob die vom Norwegischen Meteorologischen Institut vorgenommene Korrektur auf $25.4^{\circ}$ berechtigt ist. Bemerkenswert ist ein zweites Maximum von $29.5^{\circ}$ (später vom Meteorologischen Institut auf $19.5^{\circ}$ korrigiert) in der vom 2. auf den 3. Juni folgenden Nacht. Auch nächtliche Maxima sind zuvor wiederholt an der Station vorgekommen. An vergleichbaren Stationen lagen die Maxima des 2.6.1961 bei $20^{\circ} \mathrm{C}$, so daß selbst ein Maximum von $25^{\circ}$ noch auffallend hoch wäre.

\section{Tab. 1 Beobachtungen in Storslett. Einheiten: ${ }^{\circ} \mathrm{C}, \%, \mathrm{~m} / \mathrm{sec}$}

$\begin{array}{lcccccr}\text { 1961 } & Z & \text { Hauptthermometer } & \text { Minimum } & \text { Maximum } & \text { Relative Feuchtigkeit } & \text { Wind } \\ \text { 2. 6. } & 06 & 15,8 & 4,0 & 17,5 & 44 & \text { S } 2 \\ & 12 & 22,2 & - & - & 37 & \text { SSE } 5 \\ & 18 & 19,0 & 15,0 & 35,4 & 44 & \text { S } 2 \\ \text { 3. 6. } & 06 & 19,2 & 10,7 & 29,5 & 42 & \text { S 2 }\end{array}$

Die beobachteten hohen Temperaturen lassen sich nicht mit Montanföhn allein erklären, möglicherweise aber mit einer adiabatischen Absinkbewegung aus der Stratosphäre (3). Tatsächlich läßt sich aus 'Taupunktdifferenzen im Radiosondenaufstieg von Skattöra, $80 \mathrm{~km}$ westlich von Storslett, auf Absinkbewegung schließen. Am 2.6. um $00 \mathrm{Z}$ reicht diese aus der Stratosphäre bis $930 \mathrm{mb}$, der Obergrenze einer Bodeninversion. Im 12-Z-Aufstieg findet man eine Isothermie bis $940 \mathrm{mb}$, darüber bis ins 500 -mb-Niveau eine gegenüber $00 \mathrm{Z}$ um etwa $3^{\circ} \mathrm{C}$ erwärmte und auch feuchtere Luftmasse. Dies steht wohl in Zusammenhang mit aus Südwesten vordringender Warmluft. Bis zum Abend des 2.6. wird das Gebiet südlich der Linie Bodö-Helsinki von gewittrigen Schauern erfaßt (Höhendivergenz). Am 3.6. um $00 \mathrm{Z}$ zeigt die Radiosonde eine schwache Bodeninversion. Anhaltendes Absinken wird durch bis in die Stratosphäre reichende große Taupunktdifferenzen belegt. 
Auf der 500-mb-Analyse vom 2.6. $00 \mathrm{Z}$ ist ein schwacher Strahlstromast ( $50 \mathrm{kn}$ ) erkennbar, der von Südgrönland nach Schottland, zyklonal gekrümmt über Südwestnorwegen nach Jan Mayen bis Kap Tobin verläuft, weiter antizyklonal über FranzJosephs-Land nach Sibirien, wo er einen Kaltluftkern $\left(-33^{\circ}\right)$ zyklonal umströmt, während es über Nordskandinavien mit $-16^{\circ}$ bei hohem Druck relativ warm ist (Höhenkonvergenz). Am 3.6., $00 \mathrm{Z}$, zeigt die 500-mb-Karte ein stärker konzentriertes Windband, das von Nordschottland längs der norwegischen Küste bis Nordnorwegen ausgreift, dort jedoch westwärts nach Grönland umbiegt.

Für die beiden beschriebenen Temperaturmaxima in Storslett wäre demnach wohl folgende Erklärung möglich: Durch adiabatische Erwärmung beim Absinken wird der Import warmer Luft verstärkt (4a). Die in Küstennähe vorhandene Inversion wird in Storslett zumindest zeitweise weggeräumt («warme Wellen»); die Absinkbewegung greift mit sehr warmer Luft bis zum Boden durch. Überdies wirkt die zunehmende Feuchtigkeit in der mittleren Troposphäre über die atmosphärische Gegenstrahlung ebenfalls im Sinne hoher Temperaturen.

Die Situation vom 21. Mai 1961. Am Morgen des 21.5.1961 wurde in Storslett die Temperatur von $23.4^{\circ} \mathrm{C}$ beobachtet. Das Mittagsmaximum betrug nur $8^{\circ}$, doch noch um $4^{\circ}$ mehr als an zwei vergleichbaren Stationen. Die Radiosonde von Skattöra um 00 $Z$ gibt oberhalb $660 \mathrm{mb}$ bis ins 50-mb-Niveau (Ende des TEMP) große Taupunktdifferenzen an, die im 12-Z-Aufstieg auf die mittlere Troposphäre beschränkt sind und dort $15^{\circ} \mathrm{C}$ erreichen. Aus der 500-mb-Analyse von $00 \mathrm{Z}$ erkennt man einen Strahlstrom über Skandinavien, der zyklonal ein kaltes Höhentief über dem Fenno-Baltikum umfließt. Die Bodenkarte von $06 \mathrm{Z}$ läßt auf Höhendivergenz über der Halbinsel Kola und Karelien schließen. Die Annahme einer Höhenkonvergenz über Nordwestnorwegen, für die auch der angezogene Radiosondenaufstieg spricht, paßt in das Schema von der Vergenzenverteilung längs eines Strahlstrommaximums (Reiter [4b]). Auch in diesem Beispiel dürfte die adiabatische Erwärmung absinkender Stratosphärenluft für das Zustandekommen jener hohen Temperatur wesentlich sein.

Die Situation vom 12. Februar 1959. Im vorliegenden Fall wurden in ganz Finnmark hohe Temperaturen beobachtet, die Sonderstellung der Station Storslett tritt zurück, ist jedoch nicht aufgehoben.

Die zum Vergleich in 'Tabelle 2 mitaufgeführte Station Alta liegt, Storslett nicht unähnlich, im Inneren des Altafjordes auf $69^{\circ} 58^{\prime} \mathrm{N}, 23^{\circ} 22^{\prime} \mathrm{E}, 4 \mathrm{~m}$ hoch. Loppa liegt auf $70^{\circ} 20^{\prime} \mathrm{N}, 21^{\circ} 28^{\prime} \mathrm{E}, 10 \mathrm{~m}$ hoch am freien Meer. Beide Stationen sind Storslett benachbart.

Tab. 2 Wetterbeobachtungen am 12.2.1959. $\mathrm{T}=$ Luft-, $\mathrm{t}=$ Taupunkttemperatur in ${ }^{\circ} \mathrm{C}$, Wind in $\mathrm{m} / \mathrm{sec}$

\begin{tabular}{|c|c|c|c|c|c|c|c|c|c|c|c|}
\hline & $\mathrm{T}$ & $\mathrm{t}$ & $\begin{array}{c}06 \mathrm{Z} \\
\text { Minimum }\end{array}$ & Wind & $\mathrm{T}$ & $\begin{array}{l}12 \\
t\end{array}$ & $Z_{\text {Wind }}$ & $\mathrm{T}$ & $\mathrm{t}$ & $\begin{array}{c}18 \mathrm{Z} \\
\text { Maximum }\end{array}$ & Wind \\
\hline Storslett & 10 & 2 & 6 & W 2 & 10 & -4 & S 2 & 9 & -1 & 13 & SW 2 \\
\hline Alta & 6 & 2 & 0 & SSW 10 & 9 & 1 & SW 5 & 7 & 0 & 12 & S 2 \\
\hline Loppa & 7 & 4 & 5 & SW 12 & 8 & 2 & SW 10 & 5 & 3 & 9 & SW 15 \\
\hline
\end{tabular}

Minimum- sowie die 06-Z-Temperaturen sind in Storslett höher als in Alta. Beim 12-Z-Termin weisen beide Stationen hohe Temperaturen auf. Die Luft in Storslett ist besonders trocken. In den Maxima besteht nur ein geringer Unterschied. Um $18 \mathrm{Z}$ macht sich, namentlich in Loppa, Abkühlung bemerkbar. Die Morgen- und Mittagstemperaturen von Loppa sind im Hinblick auf die Lage der Stationen ziemlich hoch. Doch selbst die äußersten Küstenstationen Finnmarks erreichten Maxima von 6 bis $8^{\circ} \mathrm{C}$. Der Tagesgang durch den Sonnenstand dürfte der Jahreszeit wegen nicht erheblich sein.

Die Radiosonde von Skattöra am 12.2., $00 \mathrm{Z}$, zeigt große Feuchtigkeit bis $850 \mathrm{mb}$ aufwärts, isotherme Schichtung bis $700 \mathrm{mb}$, darüber jedoch finden sich große Tau- 
punktdifferenzen, $18^{\circ} \mathrm{C}$ im 500-mb-Niveau, was auf abwärtsgerichtete Luftbewegung schließen läßt. Dem 12-Z-Aufstieg ist zu entnehmen, daß trockene Luft sich bis auf $800 \mathrm{mb}$ herab durchgesetzt hat, die obere Troposphäre jedoch feuchter geworden ist.

Aus der 500-mb-Karte von $00 \mathrm{Z}$ erkennt man ein Band maximalen Windes, das aus dem Raume südlich Islands nach Nordost zieht und in antizyklonaler Krümmung die Küste von Nordnorwegen umläuft; eine Antizyklone liegt über dem Baltikum. Die Bodenkarte von $06 Z$ verzeichnet $Z$ yklonen bei Jan Mayen und nordöstlich von Novaja Zemlja, die Frontalzone tangiert die Bänke vor Finnmark. Niederschlag weist auf Höhendivergenz zwischen Jan Mayen und Björnöya (Bäreninsel) hin. Bis um $18 \mathrm{Z}$ hat sich der Schwerpunkt der Höhendivergenz in Richtung Nordkap verlagert.

Troposphärische Absinkbewegung unweit der Frontalzone, die wenig nördlich von Finnmark verläuft, mag die relativ hohen Temperaturen erklären.

\section{Temperaturmaxima und Ozongehalt im Februar 1959}

Der Februar 1959 zeichnete sich in Nordnorwegen durch eine unruhige Wettertype aus. An der Station Storslett traten wiederholt ungewöhnlich hohe Maxima auf. Vergleicht man den zeitlichen Gang der täglichen Temperaturmaxima von Storslett mit den Werten des Gesamtozongehaltes über Tromsö (5), so fällt auf, daß mehrfach hohen Temperaturwerten relativ großer Ozongehalt mit etwa eintägiger Phasenverschiebung folgt. Allerdings muß die Korrelation mit einem Koeffizienten von 0.26 bei 28 Wertepaaren als schwach gelten. Wie u.a. Johassex (6) fand, sind zunehmender bzw. abnehmender Ozongehalt mit Höhenkonvergenz bzw. Divergenz verknüpft. Nach $(4 \mathrm{c})$ wird winters durch die Meridionalzirkulation Ozon in hohe Breiten geführt. Absinkbewegungen im Bereich des Strahlstromsystems verstärken die Advektion von Ozon (Reiter). Man darf wohl annehmen, daß im Beispiel des Februar 1959 ein Zusammenhang zwischen Absinkbewegungen aus der Stratosphäre und hohen Temperaturmaxima besteht.

\section{FOLGERUNGEN}

Insgesamt wurden 13 Fälle ungewöhnlich hoher Temperatur in Storslett untersucht. Aus Taupunktdifferenzen vorliegender Radiosondenaufstiege, aus der Position der Bänder maximalen Höhenwindes (500-mb-Analysen) und aus der Anordnung von Niederschlagsgebieten (Höhendivergenzen) kann geschlossen werden, daß Storslett jeweils im Bereich von Höhenkonvergenzen lag. Eine Erklärung der'Temperaturwerte, die sich von denen anderer Stationen durch ihre Höhe unterscheiden, ist mit der Annahme von Montanföhn allein nur selten möglich. Das Zustandekommen jener Temperaturen dürfte ein komplexer Vorgang sein. Es erscheint wesentlich, daß dynamisch erwärmte Höhenluft zeitweise bis zum Boden vordringen kann. Die Sonderstellung von Storslett hinsichtlich hoher Temperaturen ist vielleicht der Orographie zuzuschreiben, die möglicherweise das Verdrängen der kalten Bodenluft begünstigt.

\section{LITERATURVERZEICHNIS}

1 Inspeksjonsberetning Nordreisa. V. f. N. N., Tromsö. 2 WishmaN E.: Mündliche Mitteilung. $3 \mathrm{~V}_{\mathrm{AN}}$ Mieghem: Sur l'existence de l'air tropical froid et de l'effet du foehn dans l'atmosphère libre. Mem. Inst. Roy. Mét. Belg. Nr. 12 (1939). 4 Reiter E. R.: Meteorologie der Strahlströme. Wien 1961 ; a) S. 343 b) S. 346 c) Kapitel 4. 415. 5 The Auroral Observatory at Tromsö. Observations 1959. Publikasjoner f. Det norske inst. f. kosm. fysikk. Nr. 50, Oslo 1961. 6 Johansen H.: Variations in the Total Amount of Ozone over Tromsö, and their Correlations with other Meteorological Elements. Geofys. Publikasjoner, XIX, 5 (1955).

\section{HIGH AIR TEMPERATURES IN STORSLETT (NORTHERN NORWAY)}

Strikingly high temperatures have repeatedly been observed at the meteorological station of Storslett. The station lies at times in the reach of high altitude convergencies. The origin of these high temperatures appears to be in connection with the descent of dynamically heated air to the ground. This may be due to the orography. 